\title{
El uso de psicofármacos en los centros de protección de adolescentes en Montevideo
}

\section{The use of psychoactive drugs in adolescents' residential shelters in Montevideo}

\section{O uso de psicofármacos em instituições de acolhimento institucional de adolescentes em Montevidéu}

\author{
Andrea Morteo \\ ORCID ID: 0000-0003-4169-8612 \\ Universidad de la República, Uruguay
}

Autor referente: amorteo@psico.edu.uy

Historia Editorial

Recibido: 29/03/2018

Aceptado: 12/09/2018

\section{RESUMEN}

El presente artículo estudia el fenómeno de la prescripción y el consumo de psicofármacos en los centros de protección para adolescentes, en Montevideo. Se parte del supuesto que el centro de protección incluye al dispositivo psiquiátrico como parte de su estrategia naturalizada de intervención. Se utiliza una metodología cartográfica (Passos, Kastrup \& da Escóssia, 2009), con un diseño mixto.

Por una parte, se analizan aquellas situaciones cotidianas de los centros que demandan la atención psiquiátrica y se problematiza la puesta en práctica de su tratamiento. Por otra parte, se debate acerca de la construcción de subjetividad de los jóvenes que transitan su adolescencia en estos dispositivos institucionales. Las subjetividades adolescentes aquí son comprendidas gubernamentalmente, como producto del cruce del dispositivo del centro de protección con el psiquiátrico (Foucault, 2002, 2011). El psicofármaco es, en este apartado, entendido como una tecnopolítica que en su uso sistemático, se convierte en cuerpo $y$ al hacerse inseparable, deviene en subjetividad (Preciado, 2013).

Como resultado, el $46,6 \%$ de los adolescentes tenía prescripto psicofármacos al momento de la investigación; superando los 
antecedentes relevados a nivel mundial y local. Los enunciados que fundamentan esta intervención desde la prevención y protección a los adolescentes, invisibilizan un sistema que ofrece el recurso del psicofármaco desde el inicio del proceso de institucionalización. Asimismo, se desarrollan algunas dimensiones institucionales que cronifican sus tratamientos, poniendo en cuestión la atribución exclusiva de la intervención por la situación de salud mental del joven.

Palabras clave: Centro de protección; Psicofármacos; Subjetividad; Dispositivo

\section{ABSTRACT}

This article studies the phenomenon of prescription and consumption of psychotropic drugs in adolescent residential shelters in Montevideo. It is assumed that the residential shelter includes the psychiatric device as part of its naturalized intervention strategy. A cartographic methodology is used (Passos et al., 2009), with a mixed design.

On the one hand, the daily situations of the shelters that demand psychiatric care are analyzed and the implementation of their treatment is problematized. On the other hand, there is a debate about the construction of the subjectivity of young people who live their adolescence in these institutional devices. The adolescent subjectivities here are understood governmentality, as a product of the meeting between the residential shelter device and the psychiatric one
(Foucault, 2002, 2011). Psychopharmaceutical is, in this section, understood as a techno-politics that in its systematic use, becomes a body and, as it becomes inseparable, becomes subjectivity (Preciado, 2013).

As a result, $46.6 \%$ of adolescents had psychopharmaceuticals prescribed at the time of the investigation; exceeding the global and local level surveyed. The statements of prevention and protection of adolescents that support this intervention, make invisible a system that offers the use of psychopharmaceutical from the beginning of the institutionalization process. Likewise, some institutional dimensions enunciate that their treatments are being cronify, calling into question the exclusive attribution of the intervention on mental health as a single situation of an adolescent.

Keywords: Residential shelter; Psychotropic drug; Subjectivity; Device

\section{RESUMO}

Este artigo estuda o fenômeno da prescrição e consumo de drogas psicotrópicas em instituições de acolhimento institucional para adolescentes, em Montevidéu. Presume-se que o estabelecimento de acolhimento institucional inclui ao dispositivo psiquiátrico como parte de sua estratégia de intervenção naturalizada. Uma metodologia cartográfica é utilizada (Passos et al., 2009), com um desenho misto.
Por um lado, as situações cotidianas dos centros que demandam atendimento psiquiátrico são analisadas, e a implementação de seu tratamento é problematizada. Por outro lado, há um debate sobre a construção da subjetividade dos jovens que passam sua infância e adolescência nesses dispositivos institucionais. As subjetividades adolescentes aqui são entendidas de forma governamental, como produto do cruzamento do dispositivo do acolhimento institucional 
com o psiquiátrico (Foucault, 2002, 2011). O psicofármaco é, nesta seção, entendido como uma tecnopolítica que, em seu uso sistemático, torna-se um corpo e, tornando-se inseparável, torna-se subjetividade (Preciado, 2013). Como resultado, $46,6 \%$ dos adolescentes tinham psicofármacos prescritos no momento da investigação; superando os precedentes pesquisados nos níveis global e local. As narrações que sustentam essa intervenção a partir da prevenção e proteção dos adolescentes, tornam invisível um sistema que oferece o uso de psicofármacos desde o início do processo de institucionalização. Da mesma forma, algumas dimensões institucionais encontradas cronificam seus tratamentos, colocando em questão a atribuição exclusiva da intervenção psiquiátrica como estratégia de proteção da saúde mental do jovem singular.

Palavras-chave: Acolhimento institucional; Drogas psicotrópicas; Subjetividade; Dispositivo

\section{Introducción}

LI presente artículo está compuesto por algunos resultados enunciados en la Investigación titulada La potencia de los cuerpos con psicofármacos: adolescentes en hogares de protección de tiempo completo, realizada en el marco de la Maestría de Psicología Social de la Facultad de Psicología, UdelaR. En Uruguay, la institucionalización en los centros de protección se produce como una de las principales respuestas para los niños, niñas y adolescentes (en adelante NNA) en situación de vulneración extrema de derechos. En consecuencia, Uruguay está posicionado como el país de la región que mantiene los niveles más altos de institucionalización (Comité de los Derechos del Niño, 2014). Estos centros son gestionados, directa o mediante Asociaciones Civiles, por el Instituto del Niño y del Adolescente del Uruguay (en adelante INAU).

Se parte del supuesto que el dispositivo del centro de protección incluye a la intervención psiquiátrica como parte de su estrategia naturalizada para su población adolescente. Esta investigación resulta entonces del análisis del dispositivo del centro de protección en su cruce con el dispositivo de atención psiquiátrica. A lo largo del artículo, se van desarrollando las preguntas: ¿Cómo se produce la prescripción y el consumo de psicofármacos en los centros de protección? ¿Cuáles son los motivos de 
consulta y cómo es sostenido su tratamiento? ¿Qué percepciones mantienen los adolescentes de este proceso?

Para ello, se utiliza una metodología cartográfica, con un diseño mixto (Passos et al., 2009). La estrategia metodológica comprendió tres técnicas. La primera, entrevistas en profundidad a los actores que conforman la atención en los centros de protección: educadores, directores de hogares y psiquiatras; así como también se entrevistaron a jóvenes egresados de estos centros. En segundo lugar, se relevó el porcentaje de adolescentes con prescripción de psicofármacos. Finalmente, se trianguló dicha información con un análisis documental de fuentes secundarias. Para su análisis, tomamos los aportes del análisis de contenido temático categorial (Vázquez Sixto, 1996), ordenando los relatos en significantes que abrieran diversos planos de análisis.

\section{Dialécticas en torno a la salud mental y los psicofármacos}

De acuerdo a Michel Foucault (1999), la locura o el padecimiento psíquico es comprendido como una modalidad de lo anormal; producto de una binarización y normalización previa que toma su punto de partida en los inicios del Siglo XIX. Mediante el disciplinamiento de las conductas anormales, se legitimó un saber-poder que se hizo visible en una nueva autoridad, el psiquiatra. La difusión de esta figura para Foucault, se produce fundamentalmente a partir del proceso de psiquiatrización de la infancia; concibiéndola como el lugar de fundación de toda enfermedad mental, incluso de aquella que se manifiesta recién en la vida adulta (Foucault, 2012). Asimismo, a través de la psiquiatrización del niño no loco [énfasis añadido]originalmente a través de la figura del niño idiota [énfasis añadido]-, se categorizan las anomalías entendidas como desviaciones menores; y a partir de ello se posibilita la generalización de un nuevo poder psiquiátrico. La psiquiatría para este autor, es remitida a una forma de administrar más que de intervenir terapéuticamente; y dispone 
de la posibilidad de juzgar al otro en su capacidad, y de ubicarlo en el lugar de la sinrazón (Foucault, 2012).

De acuerdo a la tesis doctoral de Andrea Bielli (2012), la psiquiatría ha desarrollado fundamentalmente dos tradiciones terapéuticas relacionadas con la forma de comprender el malestar psíquico de las personas: una más vinculada al uso de la palabra, entendiendo al malestar del sujeto como una expresión bio-psico-social; y otra tradición orgánica, donde el síntoma se comprende en última instancia como el emergente de un desequilibrio a nivel de los neurotransmisores en el organismo. Hasta la década del 50 Uruguay se encontraba en una fase pre-farmacológica; mientras que a partir de allí comienza una era farmacológica de la intervención psiquiátrica (Bielli, 2012).

Comprendidos en esta concepción organicista de la enfermedad, en la actualidad se han indagado algunos porcentajes de consumo de psicofármacos para diversas poblaciones. A modo de ejemplo, se estima que en Estados Unidos un 10\% de niños mayores de 6 años toma antidepresivos (Frances, 2014). En ese país, también un $10 \%$ de los niños de 10 años toman diariamente psicofármacos por el Trastorno por Déficit de Atención con Hiperactividad, el cual se define como uno de los criterios diagnósticos con mayor crecimiento exponencial (Frances, 2014). En consonancia, Andrea Bielli (2012) destaca en el año 2000 a los menores de 18 años como una franja donde se multiplicó un $103 \%$ el consumo de ISRS (inhibidores selectivos de recaptación de serotonina, utilizados como antidepresivos), tendencia que incluía además antipsicóticos y otros estimulantes. Asimismo, diversas investigaciones dan cuenta de que los NNA de bajos ingresos tienen mayor probabilidad de ser prescriptos con antipsicóticos; los cuales conforman un mercado en franca expansión (Angell, 2011; Frances, 2014; Míguez Passada, 2012).

Otras investigaciones nacionales nos colocan como un país que mantiene altos niveles de consumo de psicofármacos en relación a la región y a nivel mundial. 
Observatorio del Sistema Judicial de Uruguay investigó los niveles de importación de metilfenidato (Palummo, 2009), concluyendo que existe un crecimiento exponencial de la importación de la droga a partir del 2002, relacionándolo con la grave crisis socioeconómica que atravesó el Uruguay (Palummo, 2009). Otra investigación concluyó que un $30 \%$ de la infancia uruguaya consume psicofármacos prescriptos por psiquiatras u otros médicos (Míguez Passada, 2012).

A nivel institucional, el INAU realiza la segunda mayor compra nacional de psicofármacos en el 2010, principalmente por sedantes, antidepresivos y antipsicóticos (Acosta \& Scharf, 2014). En síntesis, el mismo Instituto que gestiona los centros de protección por vulneración de derechos es el que más psicofármacos compra luego del prestador de salud del Estado Uruguayo. Dentro de sus proyectos de atención a la adolescencia, se destacan los centros de privación de libertad con niveles de consumo de psicofármacos entre un 63 y 64\% (Acosta \& Scharf, 2014); es decir, los niveles más altos respecto de todas las investigaciones relevadas.

\section{Institucionalización y psicofármacos}

De la investigación se desprende que un $46,6 \%$ de los adolescentes que estaban institucionalizados en los centros de protección, se encontraban con psicofármacos prescriptos por psiquiatras al momento de la investigación. Esta cifra, no solamente supera ampliamente los niveles relevados a nivel mundial y local, sino que únicamente son superados por los hogares con medidas de seguridad para adolescentes.

¿Cómo se produce este nivel de consumo de psicofármacos en estos centros? La existencia de psicofármacos se circunscribe a la existencia de un dispositivo psiquiátrico que los prescribe, tornándolos accesibles y obligatorios (por indicación médica) para sus adolescentes. ¿Cuáles son las situaciones cotidianas de los centros de protección que culminan en una prescripción de psicofármacos? Para ello, Michel Foucault (2012) propone que el análisis del dispositivo psiquiátrico comprende al 
estudio del poder del psiquiatra, fundamentalmente a través de todos los actores que lo hacen posible en su ejercicio cotidiano (Foucault, 2012). En los centros, serían aquellos actores que lo demandan y solicitan: sus educadores y directores.

Ahora bien, es necesario describir las modalidades de atención psiquiátrica ofrecidas por el Instituto para problematizar los recorridos que los adolescentes realizan durante su proceso de institucionalización. Al ingresar al sistema de protección, el dispositivo psiquiátrico se activa para el adolescente prácticamente desde su inicio. Se colocan psiquiatras en las entrevistas de recepción, ante un nuevo ingreso. Desde la gestión de División Salud, se fundamenta en un sentido preventivo: "-No podés esperar que pase una semana, diez días... Tiene que haber un acompañamiento ahí, ¿no?... Ahí tiene que estar el dispositivo de salud mental trabajando fuerte." (Morteo, 2015, p.123). Al intervenir exclusivamente con psiquiatras como los profesionales idóneos para la atención en salud mental, se visualizó en distintos momentos un uso análogo entre los términos de atención en "salud mental" y "psiquiátrica"; generando la interrogante de la pertinencia entre un abordaje psiquiátrico como primer análisis de una situación de vulneración extrema de derechos.

Ahora bien, este joven que ingresa al sistema de Protección del INAU, es luego derivado a un hogar de ingreso mientras se procura otra solución permanente. En este primer centro, los educadores han relatado lo cotidiano y masificado del recurso del psiquiatra; enunciando con otras palabras, la intervención rápida sobre un síntoma al indicar un psicofármaco para su regulación: “-Pasaba que iba el psiquiatra a una atención y atendía, yo que sé, cuatro gurises, cinco gurises, seis gurises de una. ¿Entendés? Entonces era como tipo despacho" (Morteo, 2015, p.125). Las percepciones relevadas, tanto de educadores como de jóvenes, enuncian poco tiempo de escucha y una focalización en la indicación de psicofármacos por sobre otro tipo de abordaje clínico, más asociado al uso de la palabra. 
Posteriormente, el adolescente es trasladado a otro hogar de permanencia. Si no llega allí con una prescripción del psiquiatra, existen otros posibles recorridos institucionales. Se encuentra en División Salud del INAU, un servicio de Policlínica en Psiquiatría y un servicio de Guardia Psiquiátrica para situaciones de crisis; ambos disponibles para los centros de protección del INAU.

Respecto de la atención en la Policlínica se relevaron varios funcionamientos a destacar. En primer lugar, la frecuencia de la consulta psiquiátrica suele ser cada tres meses, ya que todos los pacientes psiquiátricos de División Salud adquieren automáticamente una hoja titulada Pauta de Crónicos que le permiten repetir medicación por ese período sin asistir a otra consulta médica. Este procedimiento está avalado en tanto hay un conocimiento de la falta de personal existente de psiquiatras y de educadores en los centros de protección. Por esta razón también, al momento de asistir a una consulta psiquiátrica, el acompañante puede ser seleccionado por ser el educador menos eficiente en el cotidiano del centro. Ello genera que muchas veces el educador acompañante desconozca el motivo de consulta o la historia de vida del joven. Los psiquiatras entrevistados entonces, manifiestan tener escasas o nulas devoluciones del hogar respecto de la evolución clínica del joven.

Siguiendo los recorridos de M. Foucault (2012), el estudio del dispositivo psiquiátrico necesita un segundo eje de análisis: la verdad del alienado como objeto de saber. En este sentido, las entrevistas realizadas con los adolescentes procuraron rescatar su verdad respecto de la atención brindada. Para ellos, la consulta es sostenida de forma poco empática con el psiquiatra tratante; sintiendo poca comprensión sobre lo que les ocurre y generando entonces, poca apertura de sí. A su vez, tanto psiquiatras, educadores y jóvenes, manifestaron que el psiquiatra suele priorizar el relato del adulto acompañante sobre el del joven, incluso cuando se visualiza al adulto desbordado en su tarea. En consecuencia, se genera un doble proceso: la angustia no-procesada por medio de un psicofármaco que la elimina y la imposibilidad 
institucional de visualizar otro tipo de terapéutica, ni en la misma consulta psiquiátrica ni con otro recurso profesional.

Ahora bien, ¿qué motivos de consulta presentan los hogares cuando solicitan un psiquiatra? Todos los actores entrevistados acuerdan que la atención psiquiátrica se conforma como respuesta institucional frente a un desajuste emocional asociado a los comportamientos derivados de la violencia. Las nominaciones más significativas que emplearon todos los adultos entrevistados fueron: violencia, incitación, agresividad, ansiedad, crisis de excitación psicomotriz y trastornos comportamentales. Sin embargo, existe un término coloquial, cotidianamente utilizado en la institución para referirse a aquellas situaciones de descontrol que ameritan convocar al psiquiatra de guardia: Descompensado [énfasis añadido]. En palabras de un educador:

Un gurí que empieza a gritar, que empieza a patalear, que se tira al piso, que capaz que eventualmente patea una puerta, que le pega a una pared... sube la escalera, baja, golpea puertas, se va, yo qué sé ¿no? Por ahí, no te hace caso... un gurí en estado de ansiedad, yo qué sé. Que lo podes controlar con un psiquiatra o podes tratar de controlarlo.... Para mí es eso "descompensado". Es lo que aprendí en INAU, después en la vida real no tengo idea qué es "descompensado" (risas). (Morteo, 2015, p. 126)

Como primer punto a destacar de este relato, la intervención del psiquiatra aparece directamente relacionada a una forma posible de control: de control de sí, pero también del control del educador sobre el joven. Es decir, parecería haber una diferencia en si el joven logra reconocer el límite marcado por el adulto a cargo o si en cambio no puede legitimarlo. Desde la perspectiva de los adolescentes, el motivo de consulta también está relacionada a la violencia: sino te podes controlar, rompes cosas, gritas e insultas. Desde su visión, la intervención con psicofármacos en comprimidos incluso resulta preventiva de un inyectable en el servicio de guardia 
psiquiátrica. Tampoco para ellos, existió otro tipo de demanda relatada que operase en paralelo al psicofármaco. En palabras de un joven:

-Claro, sino te dan es porque te podes controlar y eso... llegar al extremo de romper las cosas, de gritar de insultar...

¿`Y a vos nunca te dio por descontrolarte?

-Sí, una vuelta nomas. Pero fue porque me metieron con la cabeza al agua fría. Con un funcionario.... Fue por un problema jugando al fútbol, y no me daban bola porque yo era más chico, y entré a pegarle... a un compañero fue, y ta. Me agarraron y me metieron.... Pal agua fría. En la ducha, para que me tranquilice....

¿`Y no te llamaron al psiquiatra?

-No, me decían que me iban a llamar pero ta... yo ya me había tranquilizado y no me llamaron nada. (Morteo, 2015, p. 127)

Las categorías de conducta y control, obtuvieron aquí una forma de abordaje que consistió en brindar un acto de mayor agresividad (en tanto llevar a la fuerza a la ducha fría), donde el joven debería reconocer la autoridad de quien lo lleva. Si ello no era posible, el procedimiento indicaría un psiquiatra, remitiendo a motivos de salud mental del joven; aunque como reconoció el límite del otro, pudo evitar la consulta.

Este mecanismo de llamar al psiquiatra como figura de autoridad última (Foucault, 2012), coloca el foco de análisis en el vínculo educativo. En definitiva, los educadores son quienes sostienen sus emociones y solicitan el servicio psiquiátrico. En el análisis de sus tareas al interior de los centros, parece entenderse que la formación y cantidad de personal es insuficiente para poder diseñar estrategias de abordaje diversas frente a las angustias y las "descompensaciones" de los adolescentes; legitimando y solicitando en consecuencia, la prescripción de psicofármacos así expuesta.

La naturalización de este dispositivo se visualiza también desde la perspectiva del psiquiatra. Los mismos, han relatado cómo en sus guardias existen dificultades de 
comunicación con los educadores de los hogares, en función de las condiciones concretas de trabajo en las que se encuentran: solos en el turno a cargo de 15 adolescentes, en ocasiones con poca formación para el manejo de la angustia o con poco conocimiento de la vida del joven. En otras palabras, estos engranajes enuncian que las modalidades educativas de sostener y contener al joven repercuten en la solicitud de atención psiquiátrica. ¿Cómo trabajar las conductas relacionadas a la violencia? Un psiquiatra nos relata:

-Yo creo que la violencia tiene que ver con, y acá si creo, con la falta de posibilidades dentro del hogar, del manejo de la violencia muchas veces, ¿no? El hogar se paraliza, no hay personal suficiente, eh, hay mucho miedo a la intervención a veces policial, que a veces es necesaria. (Morteo, 2015, p.136)

Es necesario destacar que la observación del psiquiatra enuncia que el manejo de la violencia se atendería con otra figura de mayor autoridad: la policía. En este sentido, el control buscado para el manejo de estas situaciones parece estar únicamente relacionado a la puesta de límites y al manejo de la autoridad del rol del educador. Sin embargo, si la forma de manejar estos comportamientos por parte de los adultos define la convocatoria al psiquiatra, no solamente la conducta de los jóvenes debería constituirse como análisis del motivo de consulta.

Profundizando en este sentido, los adolescentes entrevistados relataron cómo la puesta de límites se produce también a partir de la amenaza [énfasis añadido] del llamado a la guardia psiquiátrica, invocándolo como un tercero que viene a poner freno a la escena. Si bien el uso del físico como puesta de límites está prohibido, la autoridad psiquiátrica y el fármaco parecen ocupar el lugar de ese otro cuerpo que viene a frenar al cuerpo adolescente. En este sentido, los jóvenes van identificando diversas modalidades educativas en torno a la solicitud del servicio psiquiátrico. La llamada al psiquiatra, parece en definitiva enmarcarse también en una lucha de poder entre el educador y el joven. Este joven relata: 
- ¿Te acordás de (educadora)? X era muy de -¡Te llamo al psiquiatra! Por eso todo el mundo también la odiaba... era buenísima igual, pero ta. Pero era muy de amenazar con eso... eso genera fricción. Y la fricción termina en caos. Dos móviles, los gurises se suben a la azotea, tiran piedras, eso. (Morteo, 2015, p. 138)

En este escenario, algunos adolescentes relataron cómo resisten a esta lucha cotidiana de poder. La "llamada al psiquiatra" puede ser utilizada "en su beneficio":

-Yo soy un chanta, un calculador. Incitaba que me llamaran al psiquiatra, cuando me llamaban al psiquiatra, me calmaba, hablaba con él. Era cualquiera (risas), hacía que se moleste el trabajo, aparte de repente destrozaba todo. Yo sabía que iban a llamar al psiquiatra, pero no iba a seguir en la misma como los otros gurises para que me pincharan.... Le rompía todo y eso los dejaba con más bronca a los educadores que son ratas; ratas, ¿viste? Que son mala gente. (Morteo, 2015, p. 138)

El último fragmento pone en evidencia que la relación entre el vínculo educativo y la solicitud del psiquiatra de guardia es además percibida por todos sus actores. El adolescente maneja esa información y la utiliza como una resistencia al dispositivo de intervención. Es necesario destacar, que si el cuestionamiento al interior del hogar se produce ante lo frecuente de la llamada al psiquiatra, es porque en algún plano no explícito se maneja que esta solicitud no se relaciona únicamente al estado mental del joven. Es decir, que incluye aspectos como la cotidianeidad, la modalidad de trabajo, los recursos humanos y subjetivos con los que cuente el educador de turno y el vínculo que establece con los adolescentes. Sin embargo, aunque todos los actores entrevistados parecen comprender estas reflexiones, el dispositivo institucional continúa funcionando sin grandes cuestionamientos formales al sistema.

Otro nivel de análisis disciplinar, permite comprender algún aspecto de este funcionamiento. De las entrevistas emergió que si el educador llama al servicio de 
guardia y el psiquiatra prescribe psicofármacos, el director del centro pierde legitimidad disciplinar para cuestionar al educador; ya que está cuestionando al mismo saber psiquiátrico. La escasa comunicación entre estos ámbitos disciplinares, genera un silencio que en definitiva culmina interviniendo únicamente sobre el joven. $Y$ de esta manera, se evita cuestionar aspectos que hacen a la tarea de cada actor y del mismo dispositivo institucional. El lugar de poder del Departamento de Psiquiatría pareciera ser incuestionable en la institucionalidad del INAU y dicho poder es sustentado también por el nivel de demanda que efectivamente tienen.

Asimismo, los educadores refieren desempeñar una tarea sumamente exigente a nivel emocional y sentirse poco acompañados en el desarrollo de las estrategias desplegadas con estos jóvenes. Parece desprenderse de la investigación, una cultura institucional que históricamente ha tenido carencias en la cantidad, formación y construcción de los equipos de trabajo encargados del cuidado directo de los jóvenes. De esta manera, el psicofármaco pareciera convertirse en un recurso institucionalizado que acalla síntomas relativos al proceso de institucionalización de un sujeto y que invisibiliza lo cotidiano de estos centros: sus dinámicas, sus técnicos y sus jóvenes como sujetos de enunciación de sí.

\section{Construcciones del sí mismo: algunas subjetividades emergentes}

El tercer y último eje de análisis del dispositivo psiquiátrico para M. Foucault (2012) es el análisis de la subjetivación producida. Con otras palabras, es necesario problematizar sobre cómo puede incidir un consumo de psicofármacos con las características descriptas, en la conformación subjetiva de un adolescente que crece en estos centros. Para este autor, son necesarias las luchas contra las formas de sujeción en tanto sumisión de la subjetividad, para alcanzar la emancipación del ser (Foucault, 1983). El estudio sobre la subjetividad es entendido aquí en su relación entre el gobierno, las formas de sujeción y sus estrategias resistenciales (Foucault, 
1983). Las resistencias visualizadas en el transcurso de la investigación, se enunciaron a través de mecanismos ocultos, que tienen por finalidad regular lo que sienten como excesivo sus jóvenes, educadores y directores de centro.

Para comenzar, los jóvenes manifestaron cómo algunos evaden tomar el psicofármaco, tirando la grajea. Los adultos encargados de su cuidado, mantienen rituales de vigilancia consecuentes: observando sus bocas al tomarla. Si esta evasiva transcurre con éxito, aparece un segundo momento de vigilancia para los adolescentes. El grupo de pares, evalúa el nivel de riesgo por no tomar el psicofármaco o lo abusivo de su prescripción. De esta manera singularizan la situación de cada joven y en función de su propio diagnóstico, le comunican al adulto o realizan un pacto de complicidad. Resulta interesante puntualizar cómo estos mecanismos juveniles mantienen criterios de salud, de autoridad y de regulación como mecanismo gubernamental en torno a sí mismos y al funcionamiento grupal (Foucault, 2011). Por un lado, es el auto-gobierno de sí -entre pares- y en este sentido se conforma como una reproducción del poder psiquiátrico (Foucault, 2012). Por otro lado, éstos parecieran encontrar un análisis particular en el uso del dispositivo psiquiátrico: no responden creyendo que toda psiquiatría es perjudicial, pero tampoco la aceptación acrítica de un sistema en el que no se sienten escuchados.

Los otros actores institucionales, algunos educadores y directores de centro, enunciaron cómo resuelven no darle medicación psiquiátrica a los adolescentes que tienen a cargo, sin poder explicitarlo dentro de la institución. Aquí la resistencia se construye devolviéndole al sujeto la capacidad reflexiva de poder dar cuenta de sí: en un acuerdo silencioso, el educador o director le pregunta al adolescente si se siente bien y si precisa la medicación; generando la decisión educativa y política de no prescribir psicofármacos ante cualquier desborde emocional.

En otro nivel de análisis, si retomamos a Michel Foucault (1983) en sus estudios acerca de las disciplinas, podríamos concluir que las consecuencias de la intervención 
psiquiátrica generan la creencia de existencia de patología mental en el joven. En este sentido, cuando los educadores deciden convocar al psiquiatra de guardia en una situación de "descompensación" de un joven, solicitan su saber para que el profesional despeje si la descompensación remite a un estado mental del joven, o si refiere a una reacción reactiva al entorno del hogar. Imaginariamente parece encontrarse que ante lo reactivo, no correspondería la prescripción de psicofármacos.

Sin embargo, los psiquiatras no parecen actuar en función de este análisis. De las entrevistas mantenidas, han manifestado que a diferencia de los hogares con medidas de seguridad, las consultas psiquiátricas se producen por una reacción sintomatológica y no por algún trastorno de tipo etiológico. Es decir, la "descompensación” parecería estar más relacionada a las conductas emergentes de la misma situación de internación, que por una patología psíquica previamente conformada.

En el correr de la investigación tampoco aparecieron instancias de intercambio entre el área de atención en salud mental y el área de los centros de protección; ni a nivel de gestión ni de atención directa. Según lo relevado, el psiquiatra suele valorar que el educador se encuentra desbordado en su tarea y debido a sus condiciones concretas laborales no comunica otras opciones de manejo de la angustia e interviene únicamente con psicofármacos; mientras el educador asienta en su imaginario, aunque con sospecha, que al prescribir psicofármacos se confirma la frágil situación de salud mental del joven. Esta dinámica institucional, imposibilita visualizar cómo las estrategias de intervención diseñadas en un área afectan directamente las dinámicas de la otra; y al escindir al sujeto en su intervención, se culmina psiquiatrizando al actor que menor poder tiene para cuestionar al mismo dispositivo de intervención.

Con el fin de problematizar sobre los efectos que produce esta dinámica institucional, se relevaron algunos enunciados que los jóvenes tienen sobre sí mismos. Al indagar las "percepciones de sí" a partir de lo devuelto en sus años de vida previos e 
institucionales, aparecieron las siguientes definiciones: "manipulador, orgulloso, calculador y "complicado de laburar" (Morteo, 2015, p. 162). Estas devoluciones fueron realizadas por otros adultos -trabajadores del INAU- y las trajeron a las entrevistas en tanto sintieron que los marcaron en la conformación de su subjetividad. Respecto de los vínculos sociales logrados durante sus tránsitos institucionales, las manifestaciones, cuando no evasivas, fueron las siguientes:

-No le pido nada a nadie, sino te gusta date media vuelta....la vida es así lamentablemente. No espero ni busco nada de nadie. Al contrario, yo de la gente espero la peor mierda. En vez de esperar una gotita de mierda, espero un camión de mierda.... Y después que pasa, después me salpica una gotita y no es nada, es bueno. Cómo yo esperaba un camión... ya no es como antes, viste? (Morteo, 2015, p. 162)

Estos últimos fragmentos representan una construcción subjetiva adolescente desde lo carente, manteniendo la expectativa de un encuentro con el otro desde la desconfianza, el ataque y la defensa.

Asimismo, en relación a la percepción de sí en torno a la medicación con psicofármacos, las respuestas de sus principales protagonistas resultaron similares:

- Allá, cuando estaba con una mina... aparecía (Educadora). ¡(Nombre), la Medicación!.... ¡Y yo la quería matar! Cuando hacía eso era para matarla. Yo después se lo cuestioné eso.... Yo me sentía re- enfermo mental, toma medicación. O mirá: $Y$ los gurises del hogar, toman medicación, todos enfermitos. (Morteo, 2015, p. 162)

En este adolescente se visualiza la tensión existente que opera entre una resistencia a pensarse como "enfermo psiquiátrico" y la producción simultánea de ese mismo sentir. Recordemos que si la cura psiquiátrica para Foucault (2012) está relacionada a un estado de total sumisión frente al poder psiquiátrico, su interiorización lo constituye como estructural de su personalidad. En otras palabras, se produce la misma 
enfermedad que en simultáneo cura; y como consecuencia, la patología trabajada únicamente con psicofármacos, genera subjetividades enfermas consecuentes. En efecto, se propone entonces dejar de entender que este sentir (de nominarse como "enfermito") debe minimizarse por ser un adolescente en crisis (Chaves, 2010) o porque no comprende los fundamentos de la psiquiatría.

La respuesta del INAU como institución social y educativa ante una situación de descontrol de un adolescente que se encuentra bajo su cuidado, debiera apuntar a la adquisición de nuevas herramientas subjetivas que le permitan un progresivo logro del control de sí y de aprendizaje de otras formas de comunicación. Con otras palabras, no debiera mantener como primer respuesta el recurrir a un objeto externo de sí para lograrlo. La tarea simbólica en la restitución del ejercicio de sus derechos, debería contemplar en el desarrollo del joven el aprendizaje del establecimiento de otros vínculos, no violentos y reparadores. Los psicofármacos gestionados de esta manera parecen anular el sentimiento de capacidad de control de sí, produciendo la necesidad de una determinada sustancia para su logro.

En relación a este fenómeno, el filósofo B. Preciado (2013) plantea que durante el siglo XX los cuerpos pasan a ser concebidos como biotipos humanos; es decir, como bienes de intercambio gestionados por las multinacionales farmacéuticas. Se produce una transformación de los conceptos de libido, conciencia, feminidad, masculinidad en realidades tangibles, en sustancias químicas y moléculas comercializables. Las subjetividades conformadas en el marco de estas sociedades, se definen por la sustancia que domina su metabolismo (sujetos cannabis, sujetos alcohol, sujetos prozac, etc.); obteniendo un cuerpo como artefacto que adquiere estatuto natural. El capitalismo actual, desde esta perspectiva, mantiene como motor el control de nuestra subjetividad y sus productos son los fármacos, las sustancias de consumo y todo lo que produce estados mentales y psicosomáticos de excitación, relajación y descarga. Este proceso de consumo, genera a la vez una sensación de control omnipotente pero 
de insatisfacción necesaria para no quedar saciado y seguir consumiendo. Es una nueva economía dominada por la era de la píldora, y su principal recurso, lo constituye el cuerpo adicto.

\section{Reflexiones finales}

El análisis realizado sobre el dispositivo psiquiátrico en el proceso de institucionalización de un adolescente en Montevideo, ha desplegado varios aspectos a resaltar. En primer lugar, que el $46,6 \%$ de los jóvenes presenten psicofármacos prescriptos, lo posicionan como el lugar donde se obtuvieron los niveles más altos de consumo de psicofármacos registrados, luego de los centros con medidas de seguridad del mismo Instituto. En consecuencia, los enunciados que fundamentan la intervención desde la prevención y protección a los adolescentes, invisibilizan un sistema que ofrece el recurso del psicofármaco ante el primer desajuste emocional y lo cronifica rápidamente por sus dinámicas de recursos humanos e institucionales.

La puesta en funcionamiento de estos dispositivos, colocan desde su inicio una situación de vulneración de derechos asociada a una necesidad de intervención psiquiátrica. Esta naturalización de la intervención psiquiátrica, invisibiliza las modalidades educativas desplegadas y las luchas de poder que suceden en los cotidianos de los centros entre los directores, educadores y jóvenes. Por otra parte, la intervención psiquiátrica sin diagnósticos relevados ni estrategias de intervención que trasciendan al psicofármaco, no conforma a ninguno de sus actores. Además, la escucha prioritaria al adulto que sí solicita medicación para el joven parece enunciar una intervención discrecional aún vigente, propia del Paradigma Tutelar de intervención sobre nuestra adolescencia.

¿Cómo pensar las subjetividades emergentes? Si en la sociedad disciplinar planteada por Michel Foucault, el control del cuerpo se producía desde el exterior, en este escenario el control se ofrece como una tecnopolítica que toma forma de cuerpo, se 
incorpora y se disuelve. Si en la dinámicas de los hogares, el fármaco se conforma como el tercer cuerpo que viene a controlar al cuerpo adolescente, este fármaco es constituido como un espacio disciplinario que habita en el cuerpo; y desde el momento que se hacen inseparables, devienen en subjetividad (Preciado, 2013). En definitiva, se desarrollan las diversas fases de un mismo y peligroso proceso por el cual se produce una intervención psiquiátrica, una prescripción de psicofármacos y un consumo deseado posterior. El cuerpo se traga al poder, a la vez que se produce un cuerpo diseñado para desearlo (Preciado, 2013).

\section{Referencias}

Acosta, V., \& Scharf, D. (2014). La ruta de las canicas: psicofármacos, niñez y adolescencia. Lento: Menores y psicofármacos. Responsabilidad estatal en la importación de medicamentos y su uso en centros de reclusión, (16), 28-34.

Angell, M. (2011). The illusions of Psychiatry. The New York Review of Books, 58(12). Recuperado de http://www.nybooks.com/articles/archives/2011/jul/14/illusionsof-psychiatry/? pagination=false \&printpage=true

Bielli, A. (2012). La introducción de los antidepresivos en Uruguay (1950-2000): Transformaciones de los saberes psicológicos. Montevideo: CISC.

Chaves, M. (2010). Jóvenes, territorios y complicidades. Una antropología de la juventud urbana. Buenos Aires: Espacio Editorial.

Comité de los Derechos del niño. (2014). Informe alternativo del cumplimiento de la Convención sobre los Derechos del Niño en Uruguay. (Informe inédito). Montevideo: s.n.

Foucault, M. (1983). El sujeto y el poder: Michel Foucault: más allá del estructuralismo y la hermenéutica. (pp. 241-259). Buenos Aires: Nueva Visión.

Foucault, M. (1999). Los anormales: Curso en Collège de France (1974-1975). Buenos Aires: Fondo de Cultura Económica. 
Foucault, M. (2002). Vigilar y castigar: Nacimiento de la prisión. Buenos Aires: Siglo $\mathrm{XXI}$.

Foucault, M. (2011). Seguridad, territorio, población: Curso en Collège de France (1977-1978). Buenos Aires: Fondo de Cultura Económica.

Foucault, M. (2012). El poder psiquiátrico: Curso en Collège de France (1973-1974). Buenos Aires: Fondo de Cultura Económica.

Frances, A. (2014). ¿Somos todos enfermos mentales? Manifiesto contra los abusos de la psiquiatría. Buenos Aires: Paidós.

Míguez Passada, M. N. (2012). La sujeción de los cuerpos dóciles. Medicación abusiva con psicofármacos en la niñez uruguaya. Buenos Aires: Estudios Sociológicos.

Morteo, A. (2015.). La potencia de los cuerpos con psicofármacos: adolescentes en hogares de protección de tiempo completo (Tesis de maestría). Universidad de la República, Facultad de Psicología, Uruguay. Recuperado de https://www.colibri.udelar.edu.uy/jspui/bitstream/123456789/4894/1/Morteo\%2c \%20Andrea.pdf

Palummo, J. (2009) Justicia y Exigibilidad de los Derechos. Análisis de las facultades, ventajas y limitaciones del Poder Judicial para incidir sobre la formulación de políticas públicas a través de un caso. Publicación del Colegio de Abogados del Uruguay, (163), 29-32.

Passos, E., Kastrup, V., \& da Escóssia, L. (2009). Pistas do Método da Cartografía. Pesquisaintervencao e producao de subjetividade. Rio de Janeiro: Sulina.

Preciado, B. (2013). Testo yonqui. Barcelona: Espasa.

Vázquez Sixto, F. (1996). El análisis de contenido temático. Barcelona: Universitat. 
Formato de citación

Morteo, A. (2018). El uso de psicofármacos en los centros de protección de adolescentes en Montevideo. Psicología, Conocimiento y Sociedad, 8(2), 118-138. doi: http://dx.doi.org/10.26864/PCS.v8.n2.7 